\title{
Nambu's Nobel Prize, the $\sigma$ meson and the mass of visible matter
}

\author{
Martin Schumachei* \\ II. Physikalisches Institut der Universität Göttingen, Friedrich-Hund-Platz 1 \\ D-37077 Göttingen, Germany
}

\begin{abstract}
The electroweak Higgs boson has been discovered in ongoing experiments at the LHC, leading to a mass of this particle of $126 \mathrm{GeV}$. This Higgs boson mediates the generation of mass for elementary particles, including the mass of elementary (current) quarks. These current-quark masses leave $98 \%$ of the mass of the atom unexplained. This large fraction is mediated by strong interaction, where instead of the Higgs boson the $\sigma$ meson is the mediating particle. Though already introduced in 1957 by Schwinger, the $\sigma$ meson has been integrated out in many theories of hadron properties because it had not been observed and was doubted to exist. With the observation of the $\sigma$ meson in recent experiments on Compton scattering by the nucleon at MAMI (Mainz) it has become timely to review the status of experimental and theoretical researches on this topic.
\end{abstract}

\section{Introduction}

Modern cosmology teaches us that matter is composed of three contributions,

(i) dark energy, amounting to $68.3 \%$ of the total matter (see e.g. [1]),

(ii) dark matter, amounting to $26.8 \%$ of the total matter (see e.g [1]).and

(iii) normal or visible matter amounting to $4.9 \%$ of the total matter.

Dark energy is related to space and has the property of expanding the universe with increasing velocity. Dark matter is observed in the dynamics of galaxies. The spiral arms of galaxies at large distances from the center move with much larger velocities than expected from Kepler's third law if only normal matter is taken into account. With additional matter which only interacts via gravitational forces Kepler's third law can be preserved. This leads to the notion of dark matter which also shows up in other phenomena, e.g. gravitational lenses.

Normal or visible matter consists of atoms, ions and electrons where the by far largest part of the mass is provided by nucleons in free space or bound in nuclei. The nucleons consist of three quarks having current-quark masses of $m_{\mathrm{u}}^{0} \sim 3-5 \mathrm{MeV}$ and $m_{\mathrm{d}}^{0} \sim 7-9$ $\mathrm{MeV}$ for the up quark and the down quark, respectively, where the ranges take into account that these masses are scale dependent. These current-quark masses are the ones which enter into the standard model and, thus, are attributed to the Higgs boson. This means that the Higgs boson is responsible for only $2 \%$ of the visible matter, whereas $98 \%$ are missing. There are different explanations for the missing $98 \%$ of the mass of the nucleon. One frequently cited explanation makes use of the total energies of the current quarks and of the gluon content of the nucleon where the nucleon mass is generated via

*mschuma3@gwdg.de 
computer simulation on the lattice (see e.g. [2]). These explanations disregard that there are much more appropriate explanations, which make use of effective theories describing the low-energy limit of QCD. These effective theories make use of the $\sigma$ meson, a low-mass sibling of the Higgs boson, which creates masses of the constituent quarks of $326 \mathrm{MeV}$ in the chiral limit, i.e. for the case that the effects of the Higgs field are turned off. Adding these masses together a nucleon mass of $\sim 980 \mathrm{MeV}$ is obtained which is increased to $\sim 1$ $\mathrm{GeV}$ after the Higgs field is turned on. This predicted nucleon mass is larger than the observed nucleon mass by $\sim 20 \mathrm{MeV}$ per constituent quark. The necessary subtraction of $\sim 20 \mathrm{MeV}$ per constituent quark corresponds to the binding energy of $\sim 8 \mathrm{MeV}$ per nucleon observed in the nucleus on the average. This tentative interpretation is reasonable because the mesonic component of the forces between constituent quarks in a nucleon and nuclear forces are of the same origin [3]. In the nucleon an additional component due to gluon exchange is expected.

The 2013 Nobel Prize in Physics has been awarded to François Englert and Peter Higgs "for the theoretical discovery of a mechanism that contributes to our understanding of the origin of mass of subatomic particles, and which recently was confirmed through the discovery of the predicted fundamental particle, by the ATLAS and CMS experiments at CERN's Large Hadron Collider". This award followed a related one where one half of the 2008 Nobel Prize in Physics was awarded to Yoichiro Nambu "for the discovery of the mechanism of spontaneous broken symmetry in subatomic physics". In order to understand the importance of this latter award it is very important to read the Nobel Lecture [4] which was presented by Giovanni Jona-Lasinio, a younger coauthor of the famous Nambu-Jona-Lasinio model [5] published in 1961. This Nobel Lecture having the title "Spontaneous symmetry breaking in particle physics: A case of cross fertilization" describes the way from superconductivity to particle physics which led to the NambuJona-Lasinio (NJL) Lagrangian

$$
\mathcal{L}=-\bar{\psi} \gamma^{\mu} \partial_{\mu} \psi+g\left[(\bar{\psi} \psi)^{2}-\Sigma_{i}\left(\bar{\psi} \gamma_{5} \boldsymbol{\tau}_{i} \psi\right)\left(\bar{\psi} \gamma_{5} \boldsymbol{\tau}_{i} \psi\right)\right]
$$

As pointed out in the Nobel Lecture this equation describes an isovector $0^{-}$pion and an isoscalar $0^{+}$. In the standard model of particle physics, the NJL model may be regarded as an effective theory for the QCD with respect to generation of the so-called constituent masses. In analogy to different descriptions of superconductivity the NJL model goes over to the linear $\sigma$ model $(\mathrm{L} \sigma \mathrm{M})$ of Gell-Mann and Lévy [6]. In the Nobel Lecture Nambu makes the important statement: "If this analogy turns out real, the Higgs field might be an effective description of the underlying dynamics." This Higgs field is the Higgs field of strong interaction represented by the $\sigma$ meson.

An explanation of the constituent-quark mass in terms of symmetry breaking mediated by the $\sigma$ meson remained uncertain as long as the $\sigma$ meson had not been observed. This, however, has changed dramatically in the last years after the $\sigma$ meson has been observed as part of the constituent-quark structure via Compton scattering by the nucleon. This experiment was carried out at MAMI (Mainz) and published in 2001 [7, 8]. The final interpretation of the results obtained required some further theoretical studies which were published in 2010 [9] , 2011 [10] and 2013 [11]. Through this experimental and theoretical work the $\sigma$ meson is by now well investigated and the process of mass generation of constituent quarks well understood. Additional aspects of the structure of the $\sigma$ meson following from $\gamma \gamma \rightarrow \pi \pi$ and $\pi \pi \rightarrow \pi \pi$ reactions are discussed in section 5.4 of Ref. [11]. 
For the Higgs boson the theoretical research started with the work of Goldstone (1961 [12] and 1962 [13] ) where it was shown that spontaneous symmetry breaking leads to massless particles in addition to a heavy scalar particle. This is not a problem for the $\sigma$ meson where the light $\pi$ mesons are massless in the chiral limit and have only a small mass as real particles, serving as pseudo-Goldstone bosons. For the Higgs boson these massless Goldstone bosons are strongly unwanted particles because they seem not to be present in nature. Therefore, in a number of papers scenarios were developed leading to symmetry breaking without Goldstone bosons. This essential modification is related to the introduction of massless gauge boson which swallow the Goldsone bosons and in this way generate mass and a longitudinal field component. In parallel to the work of Higgs [14,15] two other groups, Englert-Brout [16] and Guralnik-Hagen-Kibble [17] worked on electroweak symmetry breaking, so that - in the order of priority - the mechanism of mass generation of the electroweak gauge boson was temporarily called the EnglertBrout-Higgs - Guralnik-Hagen-Kibble mechanism. There are ongoing experiments at the LHC where the mass of the supposed Higgs boson was determined in 2012 to be 126 GeV. The related Nobel Prize was awarded in 2013 to only two researchers, Englert and Higgs, because of priority and because Brout passed away shortly before. The now official name of the mechanism is Brout-Englert-Higgs (BEH) mechanism.

Nowadays the origin of the theory of spontaneous symmetry breaking is most frequently attributed to the work of Peter Higgs [14,15, 18]. But it was Peter Higgs himself who correctly pointed out [18], that vacuum expectation values of scalar fields might play a role in breaking of symmetries was first noted by Schwinger [19]. This means that strong and electroweak symmetry breaking both can be traced back to the seminal work of Schwinger [19] and that the introduction of the $\sigma$ meson inspired the electroweak symmetry breaking, though these two processes take place at completely different scales. The interest in this interplay between the two sectors of symmetry breaking is of importance up to the present.

In the following section Schwinger's seminal ideas are described. Thereafter it is shown how the NJL model is related to the L $\sigma \mathrm{M}$. Furthermore, the formal equivalence of the $\mathrm{L} \sigma \mathrm{M}$ and the electroweak Glashow-Weinberg-Salam (GWS) theory of the standard model is briefly pointed out. This formal equivalence is complete only for the scalar Higgs $(\sigma)$ part but not for the Goldstone boson part. As stated above, in the electroweak GWS theory the Goldstone bosons do not exist as observable particles but are transferred into the longitudinal components of the electroweak gauge bosons via the $\mathrm{BEH}$ mechanism and in this way give the electroweak gauge bosons a mass. In case of the NJL and the $\mathrm{L} \sigma \mathrm{M}$ models the Goldstone bosons are identical with the triplet of $\pi$ mesons and thus are explicitly existing particles.

\section{Schwinger's $\sigma$ meson}

In 1957 Schwinger [19] was interested in the question why particles of different masses exist though from the point of view of theoretical physics a World with massless particles would be much simpler or, in other words, much more symmetric. Schwinger was aware of the fact that this question was of fundamental importance and, therefore, he gave 
his paper the title "A Theory of the Fundamental Interactions". As an abstract he cites A. Einstein with the words "The axiomatic basis of theoretical physics cannot be extracted from experiments but must be freely invented". The purpose of the paper was to present a description of the stock of elementary particles within the framework of the theory of quantized fields, which had been a subject of Schwinger's research in the years before [20]. In [19] Schwinger reconsiders the $\sigma$ meson accompanying the triplet of $\pi$ mesons, a topic which he had first discussed in [21. The important point he is making is that the absence of such a stable or metastable particle does not necessarily mean that a field of the corresponding type does not exist. If the $\sigma$ field were scalar, as contrasted with the known pseudoscalar character of the $\pi$ field, and the $\sigma$ meson had a mass greater than $2 m_{\pi}$, then the $\sigma$ meson would be highly unstable against rapid disintegration into two $\pi$ mesons. Therefore, the $\sigma$ meson has a high probability to escape experimental observation.

As a first application the mass of the $\mu$ lepton is discussed by Schwinger [19]. Within a theory of quantized fields the $\sigma$ meson corresponds to a $\sigma$ field $\phi_{(0)}$. As a field which is scalar under all operations in the three-dimensional isotopic space and in space-time, $\phi_{(0)}$ has a nonvanishing expectation value $\left\langle\phi_{(0)}\right\rangle$. Then one could at least anticipate that a suitable $\mu$-lepton mass constant might emerge from $g_{\mu}\left\langle\phi_{(0)}\right\rangle$ without requiring a particularly large coupling constant $g_{\mu}$.

Translated into present-day language Schwinger introduced a generic equation between the mass $m$ of a particle and the vacuum expectation value of a scalar field $\phi_{(0)}$ given in the form

$$
m \propto g\left\langle\phi_{(0)}\right\rangle .
$$

For the purpose of the present paper we translate Eq. (2) into three related equations, viz.

$$
\begin{aligned}
& m_{l}=g_{H l l} \frac{1}{\sqrt{2}} v, \\
& m_{q}^{0}=g_{H q q} \frac{1}{\sqrt{2}} v
\end{aligned}
$$

and

$$
m_{q}^{\mathrm{cl}}=g_{\sigma q q} f_{\pi}^{\mathrm{cl}}
$$

Eqs. (3) and (44) relate the lepton and current-quark masses, respectively, to the electroweak vacuum expectation value $v$ of the Higgs field and Eq. (5) the constituent quark mass in the chiral limit $(\mathrm{cl})$, where the effects of the Higgs field are turned off, to the pion decay constant $f_{\pi}^{c l}$ in the chiral limit. In case of Eqs. (3) and (4) the Higgs-lepton and Higgs-quark coupling constants $g_{H l l}$ and $g_{H q q}$ can be calculated from the known electroweak vacuum expectation value $v=246 \mathrm{GeV}$ and the known lepton mass $m_{l}$ and current-quark mass $m_{q}^{0}$, respectively. In case of Eq. (5) the pion decay constant in the chiral limit is $f_{\pi}^{\mathrm{cl}}=89.8 \mathrm{MeV}$ and $g_{\sigma q q}=2 \pi / \sqrt{3}=3.62$. More details will be given later.

In a second application of the $\sigma$ meson Schwinger [19] discussed the mass difference between the charged gauge bosons of weak interaction and the photon. In this work Schwinger had almost all the ingredients to construct the $\mathrm{SU}(2) \times \mathrm{U}(1)$ electroweak theory. Schwinger suggested the problem to Sheldom Glashow for further investigation. This led to the work of Sheldon Glashow [22] six years before that of Steven Weinberg [23]. 
Glashow, Weinberg and Abdus Salam [24] shared the Nobel Prize (1979) for the unification of weak interaction with electromagnetism. In this theory the masses of the three vector bosons of weak interaction are generated via the BEH mechanism, where the Goldstone bosons of electroweak symmetry breaking are transformed into the longitudinal field components and thus giving these three vector bosons a mass.

In (3) - (5) two sources of mass are discussed, viz symmetry breaking mediated by the Higgs field (Eqs. 3 and 4) and spontaneous symmetry breaking mediated by the $\sigma$ field (Eq. 51). These are the main sources of mass generation. In case of strong interaction there are in addition dynamic effects related to excited states, the interaction of spins and effects due to gluons where the latter effects show up in the form of glueballs and the $U_{A}(1)$ anomaly.

\section{Summary on the Nambu-Jona-Lasinio model and the linear $\sigma$ model}

The Lagrangians of the NJL model [5, 25 30] for the number of flavors $N_{f}=2$ are given by

$$
\begin{aligned}
& \mathcal{L}_{\mathrm{NJL}}=\bar{\psi}\left(i \not \partial-m_{0}\right) \psi+\frac{G}{2}\left[(\bar{\psi} \psi)^{2}+\left(\bar{\psi} i \gamma_{5} \boldsymbol{\tau} \psi\right)^{2}\right] \\
& \mathcal{L}_{\mathrm{NJL}}^{\prime}=\bar{\psi} i \not \partial \psi-g \bar{\psi}\left(\sigma+i \gamma_{5} \boldsymbol{\tau} \cdot \boldsymbol{\pi}\right) \psi-\frac{1}{2} \delta \mu^{2}\left(\sigma^{2}+\boldsymbol{\pi}^{2}\right)+\frac{g m_{0}}{G} \sigma \\
& G=g^{2} / \delta \mu^{2}, \quad \delta \mu^{2}=\left(m_{\sigma}^{\mathrm{cl}}\right)^{2} .
\end{aligned}
$$

In Eq. (6) the interaction between the fermions is parameterized by the four-fermion interaction constant $G$ and explicit symmetry breaking by the average current-quark mass $m_{0}=\frac{1}{2}\left(m_{u}^{0}+m_{d}^{0}\right)$. Eq. (17) differs from (6) by the fact that the interaction is described by the exchange of bosons. This leads to the occurrence of a mass counter term parameterized by $\delta \mu^{2}$. This mass counter term may be identified with the mass squared $\left(m_{\sigma}^{\mathrm{cl}}\right)^{2}$ of the $\sigma$ meson in the chiral limit. Eq. (8) shows how the two coupling constants $G$ and $g$ are related to each other.

The use of two versions of the NJL model has the advantage that for many applications the four-fermion version is more convenient whereas the bosonized version describes the interaction of the constituent quark with the QCD vacuum through the exchange of the $(\boldsymbol{\pi}, \sigma)$ meson quartet which we consider as the true description of the physical process. In the chiral limit we may write

$$
G \rightarrow \frac{g^{2}}{\left(m_{\sigma}^{\mathrm{cl}}\right)^{2}-q^{2}}, \quad q^{2} \rightarrow 0
$$

where the four-fermion coupling constant $G$ is replaced by a $\sigma$-meson propagator in the limit $q^{2} \rightarrow 0$, with $q$ being the momentum carried by the $\sigma$ meson.

The bosonized version may be obtained with the following ansatz [27]

$$
\begin{aligned}
& \sigma=-\frac{G}{g} \bar{\psi} \psi, \\
& \boldsymbol{\pi}=-\frac{G}{g} \bar{\psi} i \gamma_{5} \boldsymbol{\tau} \psi
\end{aligned}
$$


where the $\bar{q} q$ structure of the $(\boldsymbol{\pi}, \sigma)$ meson quartet is given by

$$
\left|\pi^{+}\right\rangle=u \bar{d}, \quad\left|\pi^{0}\right\rangle=\frac{1}{\sqrt{2}}(-u \bar{u}+d \bar{d}), \quad\left|\pi^{-}\right\rangle=-d \bar{u}, \quad|\sigma\rangle=\frac{1}{\sqrt{2}}(u \bar{u}+d \bar{d}) .
$$

The relation of the $q \bar{q}$ structure of the $\sigma$ meson with other structures has been investigated in [10].

The NJL model is not complete at this point and, accordingly, has to be supplemented. Using diagrammatic techniques the following equations may be found [28, 29] for the nonstrange $(\boldsymbol{\pi}, \sigma)$ sector

$$
\begin{aligned}
& M^{*}=m_{0}+8 i G N_{c} \int^{\Lambda} \frac{d^{4} p}{(2 \pi)^{4}} \frac{M^{*}}{p^{2}-M^{* 2}}, \quad M=-\frac{8 i N_{c} g^{2}}{\left(m_{\sigma}^{\mathrm{cl}}\right)^{2}} \int \frac{d^{4} p}{(2 \pi)^{4}} \frac{M}{p^{2}-M^{2}}, \\
& f_{\pi}^{2}=-4 i N_{c} M^{* 2} \int^{\Lambda} \frac{d^{4} p}{(2 \pi)^{4}} \frac{1}{\left(p^{2}-M^{* 2}\right)^{2}}, \quad f_{\pi}^{\mathrm{cl}}=-4 i N_{c} g M \int \frac{d^{4} p}{(2 \pi)^{4}} \frac{1}{\left(p^{2}-M^{2}\right)^{2}}
\end{aligned}
$$

The expression given on the l.h.s. of (13) is the gap equation with $M^{*}$ being the mass of the constituent quark with the contribution $m_{0}$ of the current quarks included. The r.h.s. shows the gap equation for the nonstrange $(n \bar{n})$ constituent-quark mass $M$ in the chiral limit. The l.h.s. of Eq. (14) represents the pion decay constant and the r.h.s. the same quantity in the chiral limit. For further details we refer to [31,32].

Making use of dimensional regularization the Delbourgo-Scadron [31] relation

$$
M=\frac{2 \pi}{\sqrt{N_{c}}} f_{\pi}^{\mathrm{cl}}, \quad N_{c}=3
$$

may be obtained from the r.h.s of Eqs. (13) and (14). This important relation shows that the mass of the constituent quark in the chiral limit and the pion decay constant in the chiral limit are proportional to each other. This relation is valid independent of the flavor content of the constituent quark, e.g. also for a constituent quark where the $d$-quark is replaced by a $s$-quark. Furthermore, it has been shown [33 35] that (15) is valid independent of the regularization scheme.

From (15) we obtain for the constituent-quark mass in the chiral limit

$$
M=326 \mathrm{MeV}, \quad m_{\sigma}^{\mathrm{cl}}=2 M=652 \mathrm{MeV}
$$

where use is made of $f_{\pi}^{\mathrm{cl}}=89.8 \mathrm{MeV}$. The mass $m_{\sigma}$ of the $\sigma$ meson including the effects of explicit symmetry breaking may be obtained from

$$
m_{\sigma}^{2}=\left(m_{\sigma}^{\mathrm{cl}}\right)^{2}+\hat{m}_{\pi}^{2}
$$

where $\hat{m}_{\pi}$ is the average pion mass. This equation has been derived in the NJL as well as in the L $\sigma \mathrm{M}$. Numerically this leads to

$$
m_{\sigma}=666 \mathrm{MeV} \text {. }
$$

As discussed in Nambu's Nobel Lecture it is of interest to compare the NJL model with the linear $\sigma$ model $(\mathrm{L} \sigma \mathrm{M})[6,36,37$. The $\mathrm{L} \sigma \mathrm{M}$ may be written in terms of three 
contributions, a fermion, a boson and an explicit contribution:

$$
\begin{aligned}
\mathcal{L}_{\mathrm{L} \sigma \mathrm{M}}^{\text {fermion }} & =\bar{\psi} i \not \partial \psi-g \bar{\psi}\left(\sigma+i \gamma_{5} \boldsymbol{\tau} \cdot \boldsymbol{\pi}\right) \psi, \\
& =\bar{\psi} i \not \partial \psi-g \bar{\psi}\left(\sigma^{\prime}+i \gamma_{5} \boldsymbol{\tau} \cdot \boldsymbol{\pi}\right) \psi+g f_{\pi}^{\mathrm{cl}} \bar{\psi} \psi, \\
\mathcal{L}_{\mathrm{L} \sigma \mathrm{M}}^{\text {boson }} & =\frac{1}{2} \partial_{\mu} \boldsymbol{\pi} \cdot \partial^{\mu} \boldsymbol{\pi}+\frac{1}{2} \partial_{\mu} \sigma \partial^{\mu} \sigma+\frac{\mu^{2}}{2}\left(\sigma^{2}+\boldsymbol{\pi}^{2}\right)-\frac{\lambda}{4}\left(\sigma^{2}+\boldsymbol{\pi}^{2}\right)^{2}, \\
\mathcal{L}_{\mathrm{L} \sigma \mathrm{M}}^{\mathrm{explicit}} & =f_{\pi}^{\mathrm{cl}} m_{\pi}^{2} \sigma .
\end{aligned}
$$

Eq. (19) is the fermion part of the L $\sigma \mathrm{M}$ Lagrangian formulated on the quark level, Eq. (20) the same relation after the shift of the $\sigma$ field

$$
\sigma^{\prime}=\sigma-f_{\pi}^{\mathrm{cl}}
$$

has been carried out. This shift leads to a mass term in the fermion part of the Lagrangian and to a constituent-quark mass in the chiral limit of

$$
M=g f_{\pi}^{\mathrm{cl}} .
$$

Eq. (24) is the same as Eq. (15) except for the difference that the NJL model provides a prediction, viz. $g=\frac{2 \pi}{\sqrt{3}}$ for the meson-quark coupling constant whereas the L $\sigma \mathrm{M}$ leaves this quantity undetermined. This is a beautiful example for the fact that the NJL model has a higher predictive power than the L $\sigma \mathrm{M}$. Explicit symmetry breaking is taken into account by the last term (22) which vanishes in the chiral limit $m_{\pi} \rightarrow 0$.

Eq. (21) describes spontaneous chiral symmetry breaking in terms of fields where a Mexican hat potential

$$
V(\sigma, \boldsymbol{\pi})=-\frac{\mu^{2}}{2}\left(\sigma^{2}+\boldsymbol{\pi}^{2}\right)+\frac{\lambda}{4}\left(\sigma^{2}+\boldsymbol{\pi}^{2}\right)^{2}
$$

is introduced, parameterized by the mass parameter $\mu^{2}>0$ and the self-coupling parameter $\lambda>0$. The minimum of the potential defines the vacuum expectation value of the $\sigma$ field $v_{\sigma}^{\mathrm{cl}}$ in the chiral limit via

$$
v_{\sigma}^{\mathrm{cl}} \equiv f_{\pi}^{\mathrm{cl}}=\sqrt{\frac{\mu^{2}}{\lambda}}
$$

There is no separate prediction for the two quantities $\mu$ and $\lambda$ and, therefore, the mass of the $\sigma$ meson in the chiral limit $m_{\sigma}^{\mathrm{cl}}=\sqrt{2} \mu$ remains undetermined. This also is a well known fact for the electroweak Higgs boson, where instead of the $\sigma$ field we have to consider the Higgs field and instead of the pions three Goldstone bosons which are absorbed into the longitudinal components of the electroweak gauge bosons. Symmetry breaking described through a Mexican hat potential is customarily named spontaneous symmetry breaking for obvious reasons. The parameter $\lambda$ of the selfinteraction term is related to the coupling constants entering into the NJL model via

$$
G=\lambda /\left(\sqrt{2} m_{\sigma}^{\mathrm{cl}}\right)^{2}, \quad g=\sqrt{\lambda / 2} .
$$

The r.h.s. relation can be derived by expressing the $\sigma$ meson mass in the chiral limit in the NJL model on the one hand and in $\mathrm{L} \sigma \mathrm{M}$ on the other.

$$
\begin{array}{ll}
\text { NJL }: & m_{\sigma}^{\mathrm{cl}}=2 g f_{\pi}^{\mathrm{cl}}, \\
\mathrm{L} \sigma \mathrm{M}: & m_{\sigma}^{\mathrm{cl}}=\sqrt{2} \mu=\sqrt{2 \lambda} f_{\pi}^{\mathrm{cl}} .
\end{array}
$$


The $S U(2)_{L} \times S U(2)_{R}$ symmetry of the $\mathrm{L} \sigma \mathrm{M}$ is explicitly broken if the potential $V(\sigma, \boldsymbol{\pi})$ is made slightly asymmetric by the addition of the explicitly symmetry breaking term given in (22). This leads to the vacuum expectation value $v_{\sigma}$ [37]

$$
v_{\sigma}=\sqrt{\frac{\mu^{2}}{\lambda}}+\frac{f_{\pi}^{\mathrm{cl}} m_{\pi}^{2}}{2 \mu^{2}}
$$

where $v_{\sigma}$ may be identified with the pion decay constant $f_{\pi}$. The mass of the $\sigma$ meson may be written in the form 36 ]

$$
m_{\sigma}^{2}=2 \lambda\left(f_{\pi}^{\mathrm{cl}}\right)^{2}+m_{\pi}^{2} .
$$

This equation (31) does not predict the $\sigma$ meson mass as long as the self-coupling parameter is undetermined. However, we have seen that the pion-quark coupling $g$ is given by

$$
g=\frac{2 \pi}{\sqrt{3}}
$$

leading to the self-coupling constant

$$
\lambda=2 g^{2}=\frac{8 \pi^{2}}{3}=26.3
$$

and to the mass parameter

$$
\mu=\sqrt{\frac{2}{3}} 2 \pi f_{\pi}^{\mathrm{cl}}=460 \mathrm{MeV} .
$$

\section{Experimental confirmation of the predicted constituent- quark and $\sigma$-meson masses}

The magnetic moment, the electromagnetic polarizabilities and the mass are fundamental structure constants of the nucleon. On a quark level these structure constants are closely related to each other. For the magnetic moment and the mass this appears to be straightforward and well known (see e.g. [38] and references therein). For the electromagnetic polarizabilities this insight is new and has been explored in detail in recent papers (see [11]). The main discovery is that the $\sigma$ meson as part of the constituent-quark structure has two important properties. On the one hand the $\sigma$ meson mediates the interaction between the three quarks in the nucleon and the $\bar{q} q$ pairs in the QCD vacuum. On the other hand the $\sigma$ meson has the property of coupling to two photons. As a consequence of this the $\sigma$ meson participates in the Compton scattering process and thus makes a dominant contribution to the electric polarizability and takes care of the total diamagnetic polarizability. Additional contributions to the Compton scattering amplitudes and the electromagnetic polarizabilities are provided by nonresonant and resonant excitation processes of the nucleon. These latter contributions can be calculated with high precision from photomeson CGLN amplitudes [39]. As a consequence it becomes possible to precisely test the predictions of the contribution the $\sigma$ meson to the Compton scattering amplitude and to the polarizabilities. This also leads to a test of the predicted structure of the $\sigma$ meson. 


\subsection{The magnetic moment of the nucleon}

It was one of the first evidences for the validity of the constituent-quark model that it allows to correctly predict magnetic moments of the nucleon. However, since there were no predictions of the constituent-quark masses these quantities were determined by adjusting the predicted magnetic moment for the proton to the experimental value. Using these constituent-quark masses for a prediction of the magnetic moment of the neutron a good but not perfect agreement with the experimental value was obtained. The remaining discrepancy observed for the neutron led to the supposition that there also should be a component due to meson exchange currents or configuration mixing in the nucleon [40].

Our procedure is different from these previous approaches and was first described in a previous preprint [40]. Our new approach makes use of the constituent-quark masses predicted by the NJL model on an absolute scale. This procedure led to an excellent agreement simultaneously for the neutron and the proton but also leaves room for some residual discrepancy of the order of $1-2 \%$ which tentatively may be attributed to meson exchange currents or configuration mixing [40]. For sake of completeness we present an update of our previous considerations.

Symmetry considerations including the color degree of freedom lead to the conclusion that the spins of identical constituent quarks are parallel to each other. Then the coupling of spin $S=1$ of the two identical constituent quarks to $S=1 / 2$ of the nonidentical constituent quark leads to two contributions to the total spin of the nucleon, as requested by the Clebsch-Gordan coefficients. Then the magnetic moments of the nucleons are given by

$$
\begin{aligned}
\mu_{p} & =\frac{4}{3} \mu_{u}-\frac{1}{3} \mu_{d}, \\
\mu_{n} & =\frac{4}{3} \mu_{d}-\frac{1}{3} \mu_{u}
\end{aligned}
$$

in units of the nuclear magneton $\mu_{N}=e \hbar / 2 m_{p}$. Constituent-quark masses enter through the relations

$$
\mu_{u}=\frac{2}{3} \frac{m_{p}}{m_{u}}, \quad \mu_{d}=-\frac{1}{3} \frac{m_{p}}{m_{d}} .
$$

On the basis of these equations we attempt a parameter-free prediction of the magnetic moments of the nucleon. From the mass of the $\sigma$ meson $m_{\sigma}=666 \mathrm{MeV}$ (18) we obtain

$$
m_{u}=\frac{1}{2} m_{\sigma}-2=331 \mathrm{MeV}, \quad m_{d}=\frac{1}{2} m_{\sigma}+2=335 \mathrm{MeV}
$$

where use is made of the fact that $m_{d}-m_{u}$ has been determined to be approximately 4 $\mathrm{MeV}$ [35]. This leads to the magnetic moments of the constituent quarks

$$
\mu_{u}=1.890, \quad \mu_{d}=-0.934
$$

and to predicted magnetic moments of the nucleon

$$
\mu_{p}^{\text {theor }}=2.831, \quad \mu_{n}^{\text {theor }}=-1.875 .
$$

Comparing these values with the experimental magnetic moments of the nucleon

$$
\mu_{p}^{\exp }=2.79285, \quad \mu_{n}^{\exp }=-1.91304
$$


we arrive at the very small differences $\Delta \mu=\mu^{\text {exp }}-\mu^{\text {theor }}$ of

$$
\Delta \mu_{p}=-0.038, \quad \Delta \mu_{n}=-0.038 \text {. }
$$

These differences are the same for the proton and the neutron and amount to $\left|(\Delta \mu / \mu)_{p}\right|=$ $1.4 \%$ and $\left|(\Delta \mu / \mu)_{n}\right|=2.0 \%$, respectively. The interesting conclusion is that the NJL model predicts constituent quark masses which lead to predictions of magnetic moments of the nucleon on a $1-2 \%$ level of agreement with the experimental values. We consider this as a first firm confirmation of the predicted constituent-quark masses. A second confirmation is provided by Compton scattering and the polarizabilities of the nucleon as described in the next subsection.

The constituent quark masses $m_{u}=331 \mathrm{MeV}$ and $m_{d}=335 \mathrm{MeV}$ are the same as the mass parameters used in other work [38, i.e. $m_{u}=330 \mathrm{MeV}$ and $m_{d}=335 \mathrm{MeV}$. The difference between our present work and [38] is that our numbers are derived on an absolute scale by applying the NJL model.

\subsection{Compton scattering and polarizabilities of the nucleon}

A nucleon in an electric field $\mathbf{E}$ and magnetic field $\mathbf{H}$ obtains an induced electric dipole moment $\mathbf{d}$ and magnetic dipole moment $\mathbf{m}$ given by

$$
\begin{aligned}
& \mathbf{d}=4 \pi \alpha \mathbf{E}, \\
& \mathbf{m}=4 \pi \beta \mathbf{H},
\end{aligned}
$$

in a unit system where the electric charge $e$ is given by $e^{2} / 4 \pi=\alpha_{e m}=1 / 137.04$. Eqs. (43) and (44) may be understood as the response of the nucleon to the fields provided by a real or virtual photon and it is evident that we need a second photon to measure the polarizabilities. This may be expressed through the relation

$$
H^{(2)}=-\frac{1}{2} 4 \pi \alpha \mathbf{E}^{2}-\frac{1}{2} 4 \pi \beta \mathbf{H}^{2}
$$

where $H^{(2)}$ is the energy change in the electromagnetic field due to the presence of the nucleon in the field.

For particles with a spin spinpolarizabilities have to be taken into account. These quantities differ from the polarizabilities by the fact that the two photons measuring these quantities are in perpendicular planes of linear polarization. In this case $\gamma_{0}$ denotes the spinpolarizability for Compton scattering in the forward direction and $\gamma_{\pi}$ the spinpolarizability for Compton scattering in the backward direction.

\subsubsection{Diagrammatic representation of Compton scattering}

The method of predicting polarizabilities is based on dispersion theory applied to partial photoabsorption cross-sections. Nevertheless, it is of interest for illustration to show the Compton scattering process in terms of diagrams as carried out in Figure 1. We see that in addition to the nonresonant single-pion channel (b) and the resonant channel (c) 


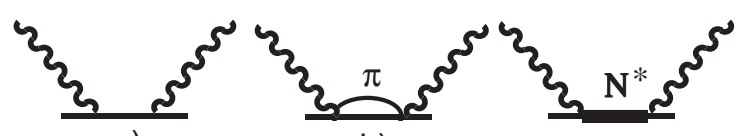

a)

b)

c)

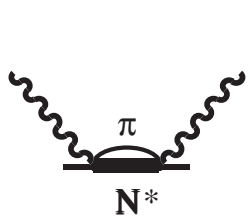

d)

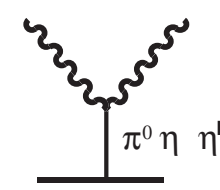

e)

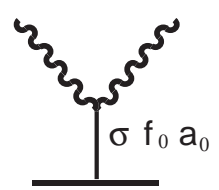

f)

Figure 1: Graphs of nucleon Compton scattering. a) Born term, b) single-pion photoproduction, c) resonant excitation of the nucleon and d) two-pion photoproduction. The crossed graphs accompanying the graphs a) to d) are not shown. The $t$-channel graphs in e) and f) correspond to pointlike singularities due to the pseudoscalar meson $\pi^{0}, \eta$ and $\eta^{\prime}$ and the scalar mesons $\sigma(600), f_{0}(980)$ and $a_{0}(980)$. These graphs are shown for illustration whereas quantitative predictions are based on dispersion theory.

we have three further contributions. Graph (d) corresponds to Compton scattering via the transition $\gamma N \rightarrow \Delta \pi$ to the intermediate state. This represents the main component of those photomeson processes where two pions are in the final state. Graph e) is the well-known pseudoscalar $t$-channel graph where in addition to the dominant $\pi^{0}$ pole contribution also the small contributions due to $\eta$ and $\eta^{\prime}$ mesons are taken into account. Graph f) depicts the scalar counterpart of the pseudoscalar pole term e). At first sight these scalar pole-terms appear inappropriate because the scalar particles and especially the $\sigma$ meson have a large width when observed on shell. However, this consideration overlooks that in a Compton scattering process the scalar particles are intermediate states of the scattering process where the broadening due to the $\pi \pi$ final state is not effective. This leads to the conclusion that the scalar pole terms indeed provide the correct description of the scattering process.

\subsubsection{Predicted polarizabilities obtained for the $s$-channel}

Using sum rules (see [11]) and applying them to photoabsorption cross sections the polarizabilities and spin polarizabilities given in Table 1 are obtained. Lines 2 contain the contributions of the $P_{33}(1232)$ nucleon resonance. This resonance is exited from the ground state via a spin-flip transition and thus is the main source of paramagnetism of the nucleon. The nonresonant electric-dipole contribution due to the $E_{0+}$ CGLN amplitude [39] is given in line 3. For the electric polarizability this corresponds to the main part of the "pion-cloud" contribution. Line 4 contains the total $s$-channel contribution. 
Table 1: Electric $(\alpha)$ and magnetic $(\beta)$ polarizabilities and spin polarizabilities for the forward $\left(\gamma_{0}\right)$ and backward $\left(\gamma_{\pi}\right)$ directions. Line 2: Resonant contributions due to the $P_{33}(1232)$ resonance. Line 3 : Single-pion nonresonant contribution due to the $E_{0+}$ CGLN amplitude [39]. Line 4: Total $s$-channel components of the polarizabilities. The electric and magnetic polarizabilities are in units of $10^{-4} \mathrm{fm}^{3}$, the spin polarizabilities in units of $10^{-4} \mathrm{fm}^{4}$.

\begin{tabular}{l|ll|ll|ll|ll}
\hline & $\alpha_{p}$ & $\beta_{p}$ & $\alpha_{n}$ & $\beta_{n}$ & $\gamma_{0}^{(p)}$ & $\gamma_{0}^{(n)}$ & $\gamma_{\pi}^{(p)}$ & $\gamma_{\pi}^{(n)}$ \\
\hline$P_{33}(1232)$ & -1.07 & +8.32 & -1.07 & +8.32 & -3.03 & -3.03 & +5.11 & +5.11 \\
$E_{0+}$ & +3.19 & -0.34 & +4.07 & -0.43 & +2.47 & +3.18 & +3.75 & +4.81 \\
$s$-channel & +4.48 & +9.44 & +5.12 & +10.07 & -0.58 & +0.38 & +8.47 & +10.00 \\
\hline \hline
\end{tabular}

\subsubsection{Contributions to the polarizabilities due to the mesonic structure of the constituent quarks}

Contributions to the polarizabilities stemming from the mesonic structure of the constituent quarks enter into dispersion theory via the $t$-channel. The main parts of these $t$-channel contributions are due to the $\pi^{0}$ meson in case of the spinpolarizability $\gamma_{\pi}$ and due to the $\sigma$ meson in case of the electromagnetic polarizabilities $\alpha$ and $\beta$. For the present article the results obtained for the $\sigma$ meson are of special interest because they contain a direct proof that the $\sigma$ meson as part of the constituent-quark structure is well understood.

The main part of the $t$-channel component of the backward spinpolarizability is given by

$$
\begin{aligned}
& \left|\pi^{0}\right\rangle=\frac{1}{\sqrt{2}}(-|u \bar{u}\rangle+|d \bar{d}\rangle), \quad \mathcal{M}\left(\pi^{0} \rightarrow \gamma \gamma\right)=\frac{\alpha_{e m} N_{c}}{\pi f_{\pi}}\left[-\left(\frac{2}{3}\right)^{2}+\left(\frac{-1}{3}\right)^{2}\right], \\
& \gamma_{\pi(p, n)}^{t}=\frac{g_{\pi^{0} N N} \mathcal{M}\left(\pi^{0} \rightarrow \gamma \gamma\right)}{2 \pi m_{\pi^{0}}^{2} m} \tau_{3}=-46.7 \tau_{3} 10^{-4} \mathrm{fm}^{4} .
\end{aligned}
$$

Analogously, we obtain for the main $t$-channel parts of the electric $(\alpha)$ and magnetic $(\beta)$ polarizabilities the relations

$$
\begin{aligned}
& |\sigma\rangle=\frac{1}{\sqrt{2}}(|u \bar{u}\rangle+|d \bar{d}\rangle), \quad \mathcal{M}(\sigma \rightarrow \gamma \gamma)=\frac{\alpha_{e m} N_{c}}{\pi f_{\pi}}\left[\left(\frac{2}{3}\right)^{2}+\left(\frac{-1}{3}\right)^{2}\right], \\
& (\alpha-\beta)_{p, n}^{t}=\frac{g_{\sigma N N} \mathcal{M}(\sigma \rightarrow \gamma \gamma)}{2 \pi m_{\sigma}^{2}}=15.210^{-4} \mathrm{fm}^{3}, \quad(\alpha+\beta)_{p, n}^{t}=0, \\
& \alpha_{p, n}^{t}=+7.610^{-4} \mathrm{fm}^{3}, \beta_{p, n}^{t}=-7.610^{-4} \mathrm{fm}^{3},
\end{aligned}
$$

where use is made of $g_{\pi N N}=g_{\sigma N N}=13.169 \pm 0.057$ [41] and $m_{\sigma}=666 \mathrm{MeV}$ as predicted by the NJL model. The sign convention used in the $q \bar{q}$ structure of the $\pi^{0}$ meson follows from [42]. It has the advantage of correctly predicting the sign of the $\pi^{0}$-pole contribution. These main contributions to the polarizabilities $\alpha, \beta$ and $\gamma_{\pi}$ have to be supplemented by the $s$-channel components and by the small components due to the scalar mesons $f_{0}(980)$ 
and $a_{0}(980)$ in case of the polarizabilities $\alpha$ and $\beta$ and due to the pseudoscalar meson $\eta$ and $\eta^{\prime}$ in case of $\gamma_{\pi}$.

The numerical evaluation of these contributions has been described in detail in previous papers [43, 44]. Here we give a summary of the final predictions and the experimental values to compare with in Table 2 and 3 .

Table 2: Backward spin polarizabilities for the proton and the neutron from [45] and Table 1

\begin{tabular}{l|ll} 
spin polarizabilities & $\gamma_{\pi}^{(p)}$ & $\gamma_{\pi}^{(n)}$ \\
\hline$\pi^{0}$ pole & -46.7 & +46.7 \\
$\eta$ pole & +1.2 & +1.2 \\
$\eta^{\prime}$ pole & +0.4 & +0.4 \\
\hline const. quark structure & -45.1 & +48.3 \\
nucleon structure & +8.5 & +10.0 \\
\hline total predicted & -36.6 & +58.3 \\
exp. result & $-(36.4 \pm 1.5)$ & $+(58.6 \pm 4.0)$ \\
\hline & unit $10^{-4} \mathrm{fm}^{4}$
\end{tabular}

Table 3: Polarizabilities for the proton and the neutron from [44] and Table 1. The total predicted values given in parentheses are obtained in case the $f_{0}(980)$ and $a_{0}(980)$ contributions are disregarded.

\begin{tabular}{l|ll|ll} 
& $\alpha_{p}$ & $\beta_{p}$ & $\alpha_{n}$ & $\beta_{n}$ \\
\hline$\sigma$ pole & +7.6 & -7.6 & +7.6 & -7.6 \\
$f_{0}$ pole & +0.3 & -0.3 & +0.3 & -0.3 \\
$a_{0}$ pole & -0.4 & +0.4 & +0.4 & -0.4 \\
\hline const. quark structure & +7.5 & -7.5 & +8.3 & -8.3 \\
nucleon structure & +4.5 & +9.4 & +5.1 & +10.1 \\
\hline total predicted & $+12.0(+12.1)$ & $+1.9(+1.8)$ & $+13.4(+12.7)$ & $+1.8(+2.5)$ \\
exp. result & $+(12.0 \pm 0.6)$ & $+(1.9 \mp 0.6)$ & $+(12.5 \pm 1.7)$ & $+(2.7 \mp 1.8)$ \\
\hline & unit $10^{-4} \mathrm{fm}^{3}$ & &
\end{tabular}

In Table 2 we find an excellent agreement between the experimental and predicted backward spin polarizabilities. This proves that both, the experimental and theoretical procedures are correct.

The most interesting feature of the polarizabilities given in Table 3 is the strong cancellation of the paramagnetic polarizabilities which is mainly due to the $P_{33}(1232)$ resonance by the diamagnetic term which is solely due to the constituent-quark structure. This constituent-quark structure component is mainly due to the $\sigma$-meson pole contribution with minor corrections due to the $f_{0}(980)$ and $a_{0}(980)$ scalar mesons. The predictions obtained for these latter contributions take into account the information we have from the 
two-photon width but remain uncertain to some extent. Therefore, it may be appropriate not to include the respective predictions into the final result. The effects of these two options may be seen in line 7 of Table 3 where the numbers in parentheses do not include the contributions from the $f_{0}(980)$ and $a_{0}(980)$ scalar mesons. Apparently the effects of the $f_{0}(980)$ and $a_{0}(980)$ scalar mesons are small and do not lead to a major uncertainty in the predictions as stated before.

A further interesting conclusion concerns the two-photon width of the $\sigma$ mesons which can be calculated from the data given in (48). Given the $\sigma$ meson mass of $666 \mathrm{MeV}$ the amplitude for the $\sigma \rightarrow \gamma \gamma$ decay for $N_{c}=3$ and $f_{\pi}=92.42 \pm 0.26 \mathrm{MeV}$ is

$$
\mathcal{M}(\sigma \rightarrow \gamma \gamma)=\frac{5 \alpha_{\mathrm{em}}}{3 \pi f_{\pi}}=0.042 \mathrm{GeV}^{-1}
$$

and the decay width

$$
\Gamma_{\gamma \gamma}=\frac{m_{\sigma}^{3}}{64 \pi}|\mathcal{M}(\sigma \rightarrow \gamma \gamma)|^{2}=2.6 \mathrm{keV}
$$

From Table 3 we obtain that the predicted and the experimental electric polarizabilities $\alpha_{p}$ of the proton are in excellent agreement with each other. Furthermore, the experimental quantity $\alpha_{p}$ has an experimental error of $\pm 5 \%$ which may be used to calculate the experimental error of the two-photon width $\Gamma_{\gamma \gamma}$ of the $\sigma$ mesons. This rather straightforward consideration leads to $\Gamma_{\gamma \gamma}=2.6 \pm 0.3 \mathrm{keV}[9]$. This result obtained from the quark model and from the electric polarizability $\alpha_{p}$ of the proton is in agreement with the two-photon width extracted from data on the $\gamma \gamma \rightarrow \pi \pi$ reaction [9], leading to a further proof that the present quark model description of the structure of the $\sigma$ meson is correct.

\subsubsection{Interference between nucleon and constituent-quark structure Comp- ton scattering}

The most direct observation of the $\sigma$ meson as part of the constituent-quark structure has been achieved via Compton scattering by the nucleon carried out in the photon energyrange between 400 and $700 \mathrm{MeV}$ at the electron accelerator MAMI (Mainz) [7, 8]. In this energy range the differential cross section for Compton scattering by the nucleon is dominated by an interference effect between a contribution from the $P_{33}(1232)$ resonance and the $\sigma$ meson pole contribution. The predicted $\sigma$ meson pole contribution depends on the $\sigma$ meson mass entering into the calculation. This means that the mass of the $\sigma$ meson can be determined by adjusting the predicted Compton differential cross-section to the experimental one.

This will be carried out in the following by investigating the energy dependence of the polarizabilities $(\alpha-\beta)$. The results obtained are shown in the left panel of Figure 2, It is clearly demonstrated that there is a constructive interference in the energy range from 400 to $700 \mathrm{MeV}$ between the contribution from the $P_{33}(1232)$ resonance and from the $t$-channel due to the $\sigma$ meson as part of the constituent-quark structure. The sum of the two contributions depends on the mass of the $\sigma$ meson adopted in the calculation and, therefore, may be used for a determination of the $\sigma$-meson mass $m_{\sigma}$ from the differential 

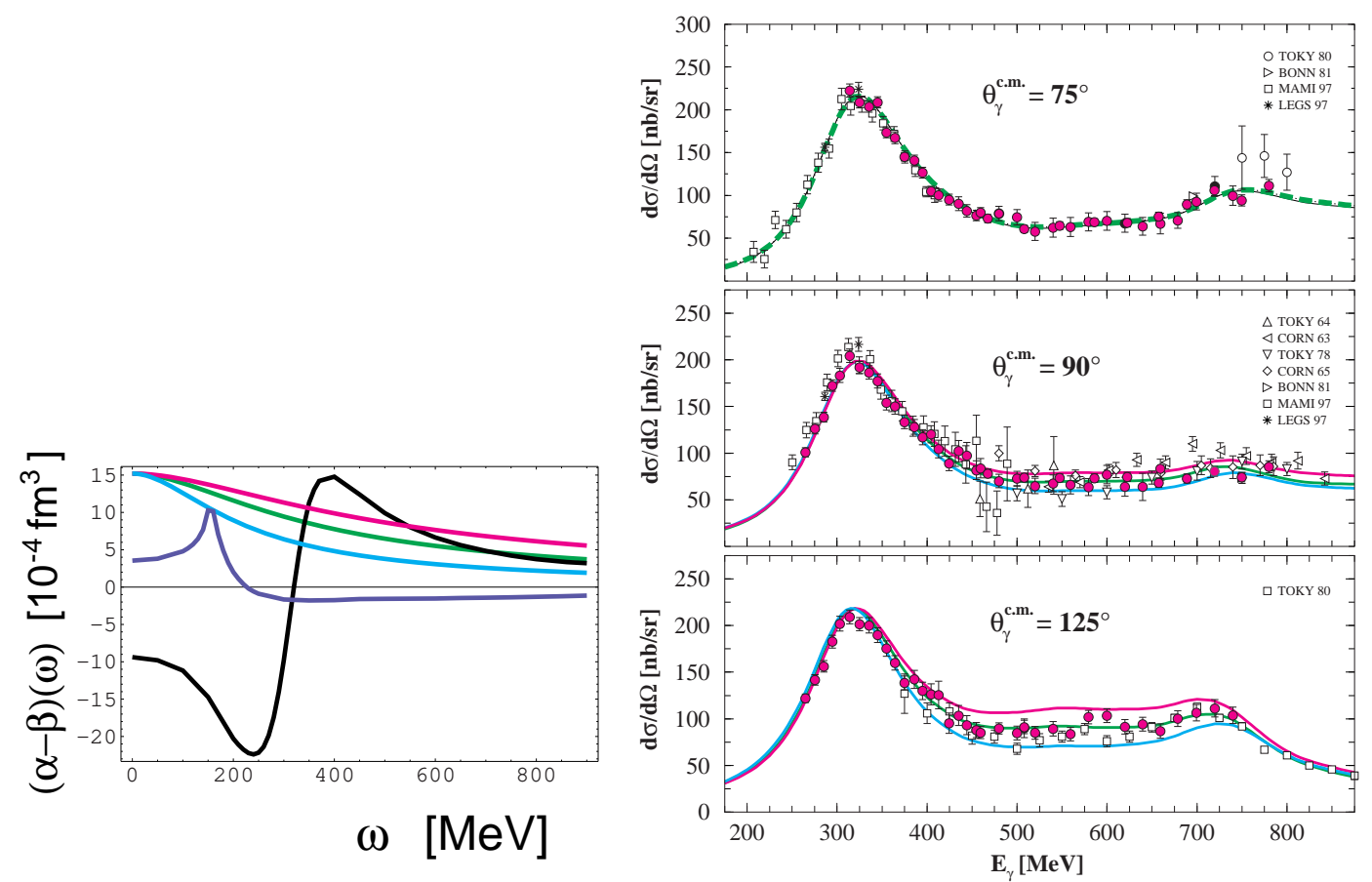

Figure 2: Left panel: Difference of generalized polarizabilities $(\alpha-\beta)(\omega)$ versus photon energy $\omega$. Solid curve starting at $(\alpha-\beta)(0)=-9.4$ : contribution of the $P_{33}(1232)$ resonance. Solid curve starting at $(\alpha-\beta)(0)=+3.5$ : Contribution of the nonresonant $E_{0+}$ amplitude. Curves starting at $(\alpha-\beta)(0)=+15.2$ : $t$-channel contribution of the $\sigma$ meson calculated for different $\sigma$-meson masses. Upper curve (dark grey or red): $m_{\sigma}=800$ $\mathrm{MeV}$. Middle curve (grey or green): $m_{\sigma}=600 \mathrm{MeV}$. Lower curve (light grey or blue): $m_{\sigma}=400 \mathrm{MeV}$. Not shown is the contribution of the $D_{13}(1520)$ resonance which cancels the $E_{0+}$ contribution in the relevant energy range from 400 to $700 \mathrm{MeV}$. Right panel: Differential cross sections for Compton scattering by the proton versus photon energy. The three panels contain data corresponding to the cm-angles of $75^{\circ}, 90^{\circ}$ and $125^{\circ}$. The three curves are calculated for different mass parameters $m_{\sigma}=800 \mathrm{MeV}$ (upper, dark grey or red), $600 \mathrm{MeV}$ (center, grey or green) and $400 \mathrm{MeV}$ (lower, light grey or blue).

cross section for Compton scattering. This is outlined in the right panel of Figure 2 where the effects of the $\sigma$-meson mass are most prominent in the backward direction, as expected. The best agreement is obtained for $m_{\sigma}=600 \pm 70 \mathrm{MeV}$, being compatible with our present knowledge of the "bare" mass of the $\sigma$ meson. As pointed out before [9] the experimental data shown in Figure 2 may be interpreted as a direct observation of the $\sigma$ meson while being part of the constituent-quark structure. Furthermore, this experiment provides us with the first and only direct determination of the "bare" mass of the $\sigma$ meson, whereas in all the other observations of the $\sigma$ meson the Breit-Wigner parameters or the position of the pole on the second Riemann sheet are determined. 


\section{The masses of nucleons and nuclei}

When we speak of the mass of visible matter we have to consider the masses of nuclei and how they are composed of nucleons, and the masses of nucleons and how they are composed of constituent quarks. Nuclei and nucleons have in common that they contain particles which are held together by interparticle forces. In case of nuclei these internucleon forces are of mesonic origin where the largest attractive part is provided by the $\sigma$ meson. In addition there are minor effects due to Coulomb forces acting between protons. In case of nucleons we have two components one mesonic component analogous to the nuclear case [3] and one gluonic component provided by the color charges of the quarks. Furthermore, minor effects due to Coulomb forces are expected. The color charges are responsible for one essential difference between nuclei and nucleons. In case of nuclei the nucleons can be extracted from the nucleus if the energy transfer is high enough. This is different in case of nucleons where the color charges lead to confinement. Single quarks cannot be extracted from the nucleon but only quark-antiquark pairs bound together to form a meson. At low energies these mesons are pions.

Except for a binding energy the mass of the nucleus is given by the masses of the nucleons. In extended nuclei the binding energies amount to $8 \mathrm{MeV}$ per nucleon on the average. For the light nuclei ${ }_{1}^{3} \mathrm{H}$ and ${ }_{2}^{3} \mathrm{He}$ the corresponding numbers are $2.83 \mathrm{MeV}$ and $2.57 \mathrm{MeV}$, respectively. These numbers are well explained in terms of mass formulae developed in nuclear physics. In order to understand the missing $98 \%$ of visible matter we, therefore, have to explain the masses of the nucleons. In the foregoing subsections we have shown that the constituent-quark masses $m_{u}=331 \mathrm{MeV}$ and $m_{d}=335 \mathrm{MeV}$ are well founded values. Without mass reduction due to the binding energy the mass of the proton would be $m_{p}^{0}=997 \mathrm{MeV}$ and the mass of the neutron $m_{n}^{0}=1001 \mathrm{MeV}$. Comparing these numbers with the experimental nucleon masses leads to the binding energies per constituent quark given in Table 4. The binding energies obtained for the quarks in the

Table 4: Binding energy $\mathrm{B}$ per constituent quark $(\mathrm{A}=3)$ in the two isospin partners p and n.

\begin{tabular}{c|c|c}
\hline nucleon & $\mathrm{p}$ & $\mathrm{n}$ \\
\hline $\mathrm{B} / \mathrm{A}$ & $19.6 \mathrm{MeV}$ & $20.5 \mathrm{MeV}$ \\
\hline
\end{tabular}

nucleon are about a factor 7.5 larger than the corresponding numbers for the nucleons in the light nuclei ${ }_{1}^{3} \mathrm{H}$ and ${ }_{3}^{3} \mathrm{He}$. This result is very plausible because of the smaller distances in the nucleon and because of gluonic components in the inter-quark forces. In a tentative attempt to understand the difference of the numbers obtained for $\mathrm{B} / \mathrm{A}$ in the proton and the neutron we write down the interquark Coulomb energy in the form

$$
U=\sum_{i, j, i<j} \frac{e_{i} e_{j}}{r_{i j}} \alpha_{\mathrm{em}} \hbar c .
$$

Then, with $\left\langle r_{i j}\right\rangle \approx 0.3 \mathrm{fm}$ we arrive at $U_{p} \approx 0 \mathrm{MeV}$ and $U_{n} \approx-1.6 \mathrm{MeV}$. This tentative consideration explains the difference of the $\mathrm{B} / \mathrm{A}$ values for the proton and the neutron as being partly due to a Coulomb attraction in the neutron. Though there is no complete 
interpretation of the $\mathrm{B} / \mathrm{A}$ values given in Table 4, the smallness of these residual mass contributions demonstrates that on the whole the masses of the nucleons are quantitatively predicted by the JNL model on a high level of precision.

\section{Discussion and conclusions}

In the foregoing we have shown that chiral symmetry breaking as provided the NJL model simultaneously explains the fundamental structure constants of the nucleon, viz. the magnetic moment, the electromagnetic polarizabilities and the mass. The magnetic moment and the electromagnetic polarizabilities are used as a proof that the predictions obtained for the constituent-quark masses are correct. These constituent-quark masses, when added together reproduce the experimental nucleon mass except for a binding energy of $\sim 20 \mathrm{MeV}$ per constituent quark. This very satisfactory result proves that the concept of mass generation as provided by the NJL model is correct.

The discovery of the electroweak Higgs boson has led to models of the vacuum where an overall Higgs field is assumed to exist. For a mass of the Higgs boson of $126 \mathrm{GeV}$ this Higgs field is expected to be elementary, i.e. not composed of fermion-antifermion pairs. The reason for this conclusion is that Higgs bosons composed of $t \bar{t}$ pairs or techniquarkantitechniquark pairs are predicted to have higher masses. In parallel to this, for the strong-interaction counterpart an overall $\sigma$ field may be assumed to exist in the QCD vacuum which is composed $u \bar{u}$ and $d \bar{d}$ pairs forming the structure $\sigma=(u \bar{u}+d \bar{d}) / \sqrt{2}$. Then the generation of the mass of the constituent quarks may be understood in terms a

$q \bar{q}$ condensate attached to the current quarks. In this condensate the $q \bar{q}$ pairs are ordered to form mesons, like the $\pi$ meson isospin triplet and the $\sigma$ meson.

\section{References}

[1] J. Beringer et al. (Particle Data Group) Phys. Rev. D 86, 010001 (2012) and 2013 partial update for the 2014 edition.

[2] L. Alvarez-Ruso, T. Ledwig, J. Martin Camalich, M.J. Vicente-Vacas, Phys. Rev. D 88, 054507 (2013), arXiv:1304.0483 [hep-ph].

[3] L. Ya. Glozman, D.O. Riska, Phys. Rep. 268, 263 (1996).

[4] Y. Nambu, Rev. Mod. Phys. 81, 1015 (2009).

[5] Y. Nambu and G. Jona-Lasinio, Phys. Rev. 122, 345 (1961); 124, 246 (1961).

[6] M. Gell-Mann, M. Levy, Nuovo. Cim. 16, 705 (1960).

[7] G. Galler et al., Phys. Lett. B 503, 245 (2001).

[8] S. Wolf et al., Eur. Phys. J A 12, 231 (2001).

[9] M. Schumacher, Eur. Phys. J. C 67, 283 (2010), arXiv:1001.0500 [hep-ph]. 
[10] M. Schumacher, J. Phys. G: Nucl. Part. Phys. 38, 083001 (2011), arXiv:1106.1015 [hep-ph].

[11] M. Schumacher, M.D. Scadron, Fortschr. Phys. 61, 703 (2013), arXiv:1301.1567 [hep$\mathrm{ph}$.

[12] J. Goldstone, Il Nuovo Cim. 19, 154 (1961).

[13] J. Goldstone, A. Salam, S. Weinberg, Phys. Rev. 127, 965 (1962).

[14] P.W. Higgs, Phys. Lett. 12, 132 (1964).

[15] P.W. Higgs, Phys. Rev. Lett. 13, 508 (1964).

[16] F. Englert, R. Brout, Phys. Rev. Lett. 13, 321 (1964).

[17] G.S. Guralnik, C.R. Hagen, T.W.B. Kibble, Phys. Rev. Lett. 13, 585 (1964).

[18] P.W. Higgs, Phys. Rev. 145, 1156 (1966).

[19] J. Schwinger, Ann. Phys. (N.Y.) 2, 407 (1957).

[20] J. Schwinger, Phys. Rev. 82, 914 (1951); 91, 713 (1953); 91, 728 (1953); 92, 1283 (1953); 93, 615 (1954); 94, 1362 (1954).

[21] J. Schwinger, Phys. Rev. 104, 1164 (1956).

[22] S. Glashow, Nucl. Phys. 22, 579 (1961).

[23] S. Weinberg, Phys. Rev. Lett. 191264 (1967).

[24] A. Salam, in Elementary Particle Theory, N. Svartholm, eds. Amqvist and Wiksells, Stockholm (1969) p. 376.

[25] D. Lurié, A.J. MacFarlane, Phys. Rev. B 136, 816 (1964).

[26] T. Eguchi, Phys. Rev. D 14, 2755 (1976); 17, 611 (1978).

[27] U. Vogl and W. Weise, Prog. Part. Nucl. Phys. 27, 195 (1991).

[28] S.P. Klevansky, Rev. Mod. Phys. 64, 649 (1992).

[29] T. Hatsuda. T. Kunihiro, Physics Reports 247, 221 (1994).

[30] J. Bijnens, Phys. Rep. 265, 369 (1996).

[31] R. Delbourgo, M. Scadron, Mod. Phys. Lett. A 10, 251 (1995), arXiv:hep-ph/9910242; Int. J. Mod. Phys. A 13, 657 (1998), arXiv:hep-ph/9807504.

[32] M. Schumacher, Eur. Phys. J. A 30, 413 (2006). Eur, Phys. J. A 32, 121 (2007) (E), arXiv:hep-ph/0609040.

[33] R. Delbourgo, A.A. Rawlinson, M.D. Scadron, Mod. Phys. Lett. A 13, 1893 (1998), arXiv:hep-ph/9807505. 
[34] R. Delbourgo, M.D. Scadron, Mod. Phys. Lett. A 17 (2002) 209, arXiv:hep-ph/0202104.

[35] M.D, Scadron, G. Rupp, R. Delbourgo, Fortschr. Phys. 61, 994 (2013), arXiv:1309.5041 [hep-ph].

[36] V. De Alfaro, S. Fubini, G. Furlan, C. Rossetti, Currents in Hadron Physics, North Holland, Amsterdam 1973.

[37] J. F. Donoghue, E. Golowich, B.R. Holstein Dynamics of the Standard Model Cambridge Monograph on Particle Physics, Cambridge University Press 1996.

[38] Z. Ghalenovi, A.A. Rajabi, S. Qin, D.H. Rischke, arXix:1403.4582 [hep-ph].

[39] D. Drechsel, S.S. Kamalov, L. Tiator, Eur. Phys. J. 34, 69 (2007), arXiv:0710.0306 [nucl-th].

[40] M. Schumacher, arXiv:0805.2823 [hep-ph].

[41] D.V. Bugg, Eur. Phys. J. C 33 (2004) 505.

[42] F.E. Close, An Introduction to Quarks and Partons, Academic Press, London, 1979.

[43] M. Schumacher, Eur. Phys. J. A 34, 293 (2007), arXiv:0712.1417 [hep-ph].

[44] M. Schumacher, Nucl. Phys. A 826, 131 (2009), arXiv:0905.4363 [hep-ph].

[45] M. Schumacher, M.I. Levchuk, Nucl. Phys. A 858, 48 (2011), arXiv:1104.3721 [hepph]. 